\title{
Risk Acceptance and Expectations of Scalp Allotransplantation
}

Jun Ho Choi ${ }^{1}$, Kwang Seog Kim', Jun Ho Shin', Jae Ha Hwang ${ }^{1}$, Sam Yong Lee ${ }^{1}$

Departments of ${ }^{1}$ Plastic and Reconstructive Surgery and ${ }^{2}$ Preventive Medicine, Chonnam National University Medical School, Gwangju, Korea
No potential conflict of interest relevant to this article was reported.

\begin{abstract}
Background: In scalp allotransplantation, the scalp from a brain-dead donor, including hair, is transferred to a recipient with scalp defects. Opinions differ on the appropriateness of scalp allotransplantation. In order to maintain graft function and cosmetic outcomes, scalp transplantation recipients would need to receive lifelong immunosuppression treatments. The risks of this immunosuppression have to be balanced against the fact that receiving a scalp allotransplant does not extend lifespan or restore a physical function. Therefore, the present study aimed to investigate risk acceptance and expectations regarding scalp allotransplantation in different populations.

Methods: A questionnaire survey study was conducted. A total of 300 subjects participated; survey was conducted amongst the general public $(n=100)$, kidney transplantation recipients $(n=50)$, a group of patient who required scalp reconstruction due to tumor or trauma $(n=50)$, and physicians $(n=100)$. The survey was modified by using the Korean version of the Louisville instrument for transplantation questionnaire.

Results: Risk acceptance and expectations for scalp transplantation varied widely across the groups. Kidney transplantation recipients revealed the highest risk acceptance and expectations, whereas the physicians were most resistant to the risks of scalp transplantation.

Conclusion: Our study demonstrates that, in specific groups, scalp allotransplantation and the need for immunosuppression carries an acceptable risk despite the lack of lifeextending benefits. Our results suggest that scalp allotransplantation can be an acceptable alternative to existing scalp reconstruction surgeries in patients with pre-existing need for immunosuppression.
\end{abstract}

Keywords: Scalp / Vascularized composite allotransplantation / Surveys and questionnaires / Reconstructive surgical procedures / Patient acceptance of health care

\section{INTRODUCTION}

Scalp allotransplantation refers to transfer of scalp tissues from brain-dead donors to living recipients. Scalp defects can result from tumor resection, trauma, and burn injuries. To reconstruct scalp defects, various reconstructive methods have been used, including flap operations, skin grafts, and tissue expansion. Howev-

\footnotetext{
Correspondence: Kwang Seog Kim

Department of Plastic and Reconstructive Surgery, Chonnam National University Medical School, 42 Jebong-ro, Dong-gu, Gwangju 61469, Korea

E-mail: pskim@chonnam.ac.kr

*This article was presented at the 73rd Congress of the Korean Society of Plastic and Reconstructive Surgeons on November, 2015 in Seoul, Korea.

Received May 2, 2016 / Revised May 24, 2016 / Accepted May 25, 2016
}

er, traditional methods of scalp reconstruction have many limitations. Therefore, scalp allotransplantation could be a promising alternative.

Tissue expansion can be used to reconstruct the scalp using the uninjured portion of the remaining scalp. This approach does not work; however, in defects involving greater than $50 \%$ of the scalp by area. Free-flap operations can reconstruct such large scalp defects, but the cosmetic outcome is unsatisfactory because the free flap will not match in skin texture, color, and hair growth.

Scalp allotransplantation is a composite tissue allotransplantation, including the hair of donors. The operation has been reported in just two cases in the literature. The first case was between 
monozygotic twins who had no need for immunosuppressive therapy, in which two flap operations were performed based on the superficial temporal artery. The transplant was successful and hair growth from the flap was observed after long-term monitoring [1]. The other case was that of a 72-year-old woman with stage IIIC recurrent cutaneous malignant melanoma of the parietal scalp. The patient was treated with a wide excision, including part of the scalp, the face, the neck, and both ears. The resulting defect was reconstructed using a composite tissue allotransplantation, including both ears and the scalp from a young man who had been declared brain dead [2].

Patients who undergo scalp allotransplantations need continuous immunosuppressive therapy that can pose life-threatening complications. Unlike other organ transplants such as kidney or liver, the functional or cosmetic improvement due to a scalp transplant does not extend a patient's life. Thus, scalp allotransplantation raises ethical questions [3]. Furthermore, patients may have ethical objections or experience mental distress due to the fact that the scalp was removed from a brain-dead person [4]. Therefore, it is important to objectively measure and evaluate the level of risks and benefits of composite tissue allotransplantation before actual application.

Recent studies have reported gaps in the knowledge regarding risk acceptance and expectations for composite tissue allotransplantation between experts and non-experts in a number of countries. In South Korea, similar studies have been conducted to evaluate risk acceptance and expectations for allotransplantation of the face, hands, feet, and larynx [5-7]. However, no such survey study has been performed on scalp allotransplantation within South Korea.

\section{METHODS}

\section{Samples}

The questionnaire study incorporated four subpopulations within South Korea, to evaluate risk acceptance and expectations for scalp allotransplantation. The first group consisted of relatives of patients who had minimal expertise. The second group was composed of kidney transplantation recipients who were receiving lifelong immunosuppressive therapy. The third group comprised patients who had undergone scalp reconstruction with non-hair bearing tissues. The fourth group comprised resident doctors who performed surgery and had expert medical knowledge and clinical experience. The study protocol was reviewed and authorized by the Institutional Review Board of Chonnam National University Hospital.

\section{General public group}

The author conducted the survey to 100 individuals who came with their family members to the outpatient department of our institute between March 2013 and April 2015. The participants ranged in age from 23 to 75 years (mean age, $44.8 \pm 13.3$ years).

\section{Kidney transplantation recipients group}

The kidney transplantation recipients at our institution were given the same questionnaire survey $(\mathrm{n}=50)$. The patients in this group were between 32- year-old and 64-year-old (mean age, 48.9 \pm 8.2 years).

\section{Post-scalp reconstruction patients group}

The third group consisted of patients who had undergone reconstruction of scalp defect from tumor excision or trauma between December 2007 and November 2014. Of the 50 patients completing survey, free flap operation had been performed in 2 patients, with the 48 remaining patients having received skin grafts. The scalp defect sizes ranged from 4 to $170 \mathrm{~cm}^{2}$, and the causes of tissue defect was trauma in 24 cases and tumor resection in 26 cases. The patients were between 29 and 80 years of age (mean age, 60.6 \pm 10.7 years).

\section{Doctor group}

The survey was administered to a group of 100 resident doctors working at our institute, with ages ranging from 26 to 36 years (mean age, $31.0 \pm 2.8$ years).

\section{Survey method}

Barker et al., provided the potential scenarios for allotransplantation of the face, hands, feet, kidney, and larynx, and developed the 
Louisville instrument for transplantation (LIFT), which is a survey tool used to objectively evaluate the degree of risk acceptable in relation to the potential benefits through composite tissue allotransplantation [5].

Kim et al. [8] translated the English version of the LIFT into the Korean language with the help of an expert translator, and then investigated the reliability and validity of the LIFT questionnaire, as well as risk acceptance and expectations of allotransplantation of the face, hands, feet, and larynx. In the present study, the same method was used for studying risk acceptance and expectations for scalp allotransplantation.

Evaluators were plastic surgery residents, who explained the technical, ethical, and social aspects of scalp allotransplantation to participants before the questionnaire was provided. The translated LIFT questionnaire was completed by the participants. The evaluators were allowed to assist in explaining medical concepts to the lay persons to allow for revision of answers.

To help with understanding the potential need for scalp allotransplantation, participants were provided with photographs of various scalp defects and non-allotransplantation reconstruction outcomes, to help them understand that traditional scalp defect reconstruction results in an unnatural appearance. The adverse reactions caused by taking immunosuppressive agents after surgery were also explained to the participants. The participants also understood that, if tissue rejection occurred after scalp allotrans- plantation, reoperation with autologous tissue without hair would be necessary.

\section{Survey contents}

\section{Risk acceptance of scalp allotransplantation}

Risk acceptance is a concept representing the degree of risk that can be accepted in return for particular benefits. The present study investigated two aspects of risk acceptance: adverse reactions to immunosuppression and tissue rejection risks.

\section{Risk acceptance of the side effects of immunosuppression}

Patients were advised that adverse reactions of immunosuppression can range from relatively minor symptoms such as nausea, vomiting, diarrhea, constipation, weight gain, dizziness, and headache to serious complications such as infection, tumor, hypertension, diabetes, and toxicity to peripheral organs. They were also notified that in case of severe tissue rejection, the graft would have to be removed. They were instructed to express their intention to receive scalp allotransplantation by responding "yes" or "no" after considering the adverse reaction risk from immunosuppression. Assuming that the remaining lifespan would be 10 years, the length of lifespan they would be willing to give up due to adverse reactions to immunosuppression was also investigated (Fig. 1).

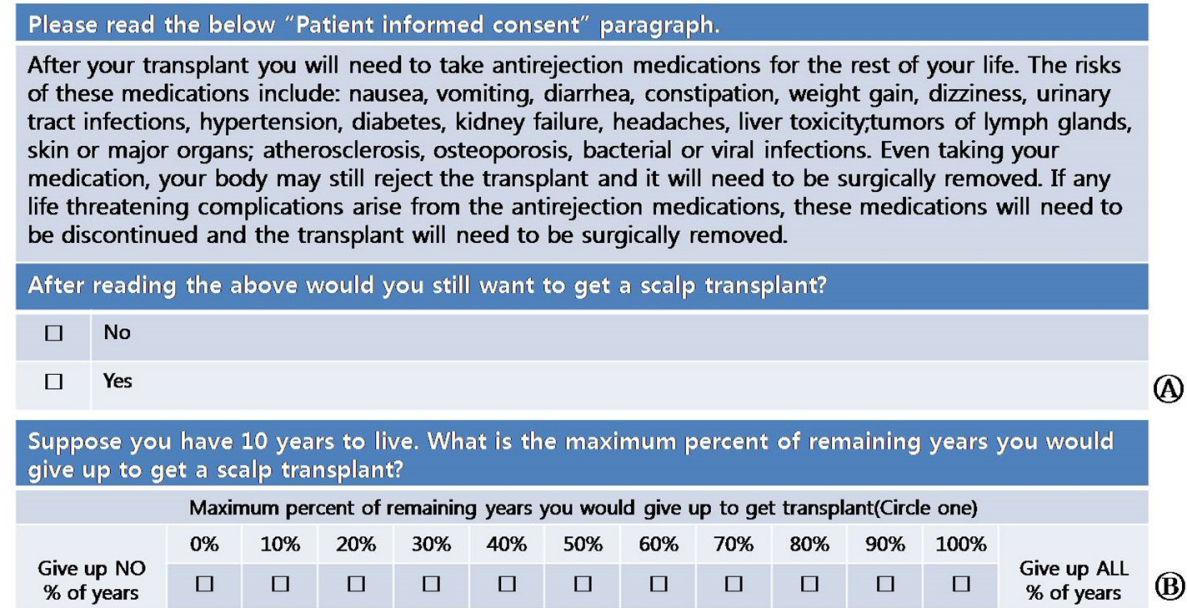

Fig. 1. Questionnaire focused on risk acceptance of immunosuppression. (A) Question inquiring about acceptance of immunosuppression side effects. (B) Question inquiring about maximum immunosuppression risk acceptability. 


\section{Risk acceptance of tissue rejection}

When the risk of rejection within 1 year of scalp allotransplantation was assumed to be $50 \%$, they were instructed to express their intentions to receive transplantation by "yes" or "no" responses, and were also requested to indicate the maximum degree of risk for tissue rejection acceptable for them to still undergo scalp allotransplantation (a higher number meant greater intention to accept risk) (Fig. 2).

\section{Expectations of scalp allotransplantation}

\section{Expectations of quality of life}

For assessment of quality of life expectations, participants were requested to score the quality of life they assumed they would have because of the unpleasant appearance, or 'ugliness', of hairless scalp, and the change in quality of life after scalp allotransplantation. They were asked to answer using Arabic numerals from 0 to 10 , where the worst condition was represented by a ' 0 ' and the best condition was represented by a '10'. In order to avoid confusion on what constitutes ugliness of a hairless scalp, participants were also given pre/postoperative photographs of the patient who had scalp allotransplantation to the parietal area for stage IIIC recurrent cutaneous malignant melanoma (Fig. 3).

\section{Functional improvement after transplantation and im- portance of function and appearance}

When it was assumed that the remaining lifespan would be decreased by $1 / 3$ due to adverse reactions of the immunosuppression treatment, each group indicated the minimum degree of functional improvement for which they could accept the treatment. They were asked to select a number from 0 to 100, by which a higher number meant a higher improvement.

In addition, participants were asked to indicate the importance of function and appearance of the scalp to be transplanted with a

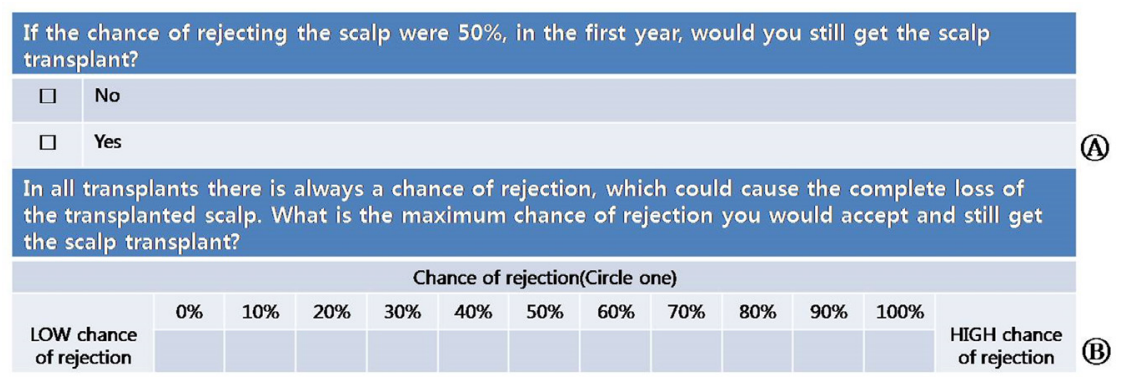

Fig. 2. Questionnaire focused on risk acceptance of rejection. (A) Question inquiring about acceptance of 50\% chance of tissue rejection. (B) Question inquiring about maxi $\neg$ mum rejection risk acceptability.

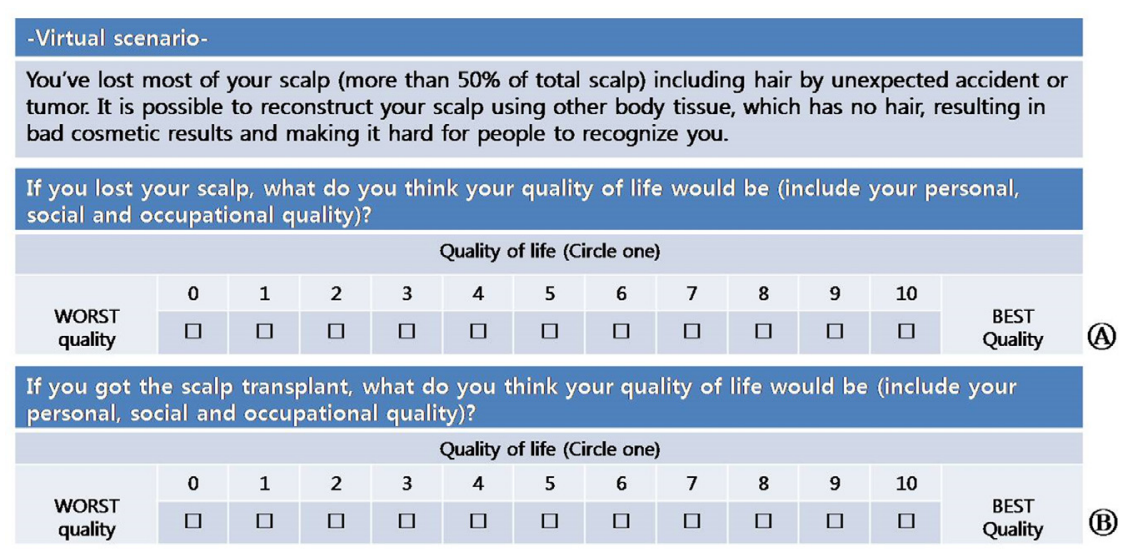

Fig. 3. Questionnaire focused on quality of life. (A) Question inquiring about quality of life if the larynx is destroyed. (B) Question inquiring about expected quality of life after transplantation. 
number from 0 to 10 , in which a higher number meant more important (Fig. 4).

\section{Statistical analysis}

Collected data were recorded and summarized using Excel (Microsoft Office Excel 2010, Microsoft Corp, Redmond, WA, USA). Statistical analysis was performed using the statistical package SPSS (SPSS ver. 15.0, SPSS Inc., Chicago, IL, USA). One-way analysis of variance was conducted to compare the differences between groups, post hoc multiple comparisons were performed to identify areas of clear differences, and a chi-square test was used for analysis of dichotomous risk acceptance survey questions. All the significance levels were set to less than $5 \%$.

\section{RESULTS}

\section{Examination of risk acceptance of scalp allo- transplantation}

\section{Risk acceptance of the side effects of immuno- suppression}

Considering adverse reactions to immunosuppression, $86 \%$ of the general public and $92 \%$ of the group who underwent kidney transplantation responded positively to scalp allotransplantation, which was higher than the proportion in the group that had undergone reconstruction for defects on the scalp (60\%) and the doctor group (68\%). With the assumption that the expected lifespan was only 10 years, the maximum percentages of lifespan that participants would give up after scalp allotransplantation surgery was $31.4 \%$ for the general public group, $41.6 \%$ for the kidney transplantation group, $24.4 \%$ for scalp reconstruction group, and 20.7\% for the doctor group.

One-way analysis of variance found significant differences among the four groups $(p<0.001)$. However, the post hoc multiple comparison found no clear difference between the scalp reconstruction group and the doctor group ( $p=0.611$ ) (Fig. 5).

\section{Risk acceptance of tissue rejection}

Assuming a 50\% risk of rejection in the first year after scalp allotransplantation, $69 \%$ of the general public group and $74 \%$ of the group with kidney transplantation answered 'yes' to having scalp allotransplantation, which were higher than those of the scalp reconstruction group (40\%) and the doctor group (55\%) $(p<0.001)$.

Furthermore, the maximum acceptable risk of tissue rejection was $44.7 \%$ for the general public group and $48.6 \%$ for the group with kidney transplantation, which was significantly different from the other two groups $(p<0.001)$. There was no significant difference between the general public group (44.7\%) and kidney transplantation group $(48.6 \%)(p=0.561)$ nor between the scalp re-

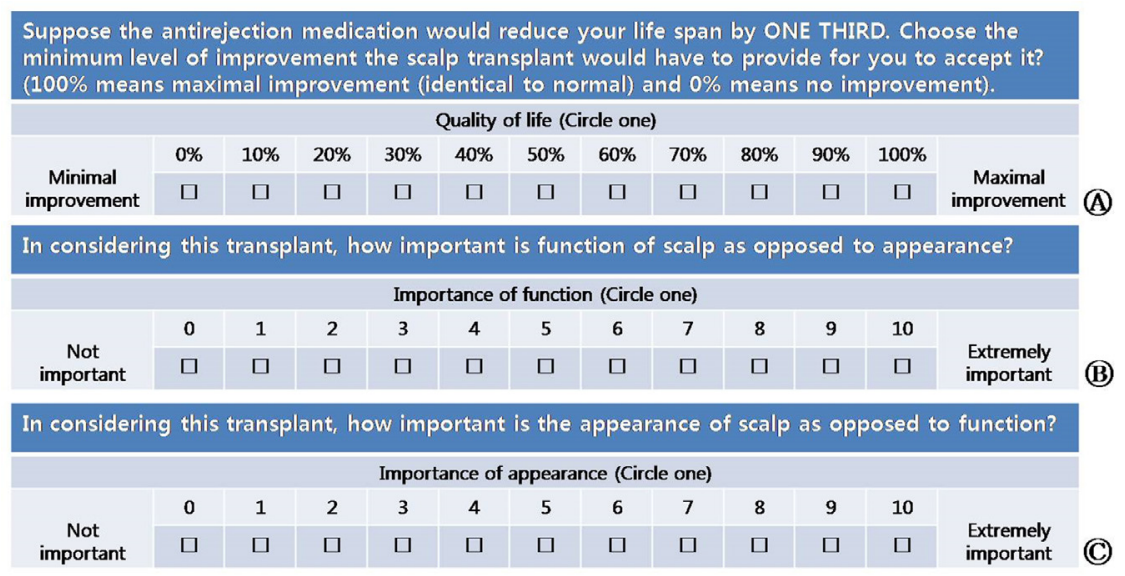

Fig. 4. Questionnaire focused on expectations of functional and aesthetic outcomes. (A) Question inquiring about expected function after transplantation. (B) Question inquiring about importance of function. (C) Question inquiring about importance of appearance. 
construction group (30.2\%) and the doctor group (31.9\%) $(p=0.941)($ Fig. 5).

\section{Examination of group expectations of scalp allotransplantation}

\section{Quality of life}

Quality of life after hairless reconstruction was given the following scores: 3.1 for the general public group, 3.4 for the group with kidney transplantation, 2.3 for the scalp reconstruction group, and 2.2 for the doctor group $(p<0.001)$. Quality of life after scalp allotransplantation were given scores of $8.3,8.4,7.4$, and 7.5 for the general public group, the group that underwent kidney transplantation, the group that had undergone reconstruction for defects on the scalp, and the doctor group, respectively. While the quality of life scores differed across all four groups (all $p<0.05$ ), the most significant difference was found in comparisons between the doctors and the general public group $(p=0.003)$ (Fig. 6).

\section{Expected function after transplantation}

Assuming immunosuppressive drugs would reduce the remaining life span by $1 / 3$, reduction of the remaining lifespan was acceptable to $74 \%$ of the general public, $75 \%$ of the kidney transplan- tation group, $73 \%$ of the scalp reconstruction group, and $68 \%$ of the doctor group, as long as the transplanted scalp was fully functional $(p=0.117)$ (Fig. 6).

\section{Importance of function and appearance}

The importance of improvement in appearance of the scalp after scalp allotransplantation was rated 7.7, 7.9, 7.3, and 6.6 by the general public group, the group with kidney transplantation, the scalp reconstruction group, and the doctor group, respectively $(p<0.001)$. The importance of normal function of the scalp in comparison to appearance was rated 7.0, 7.0, 6.9, and 5.7 by the general public group, the group with kidney transplantation, the scalp reconstruction group, and the doctor group, respectively $(p<0.001)$.

\section{DISCUSSION}

Although scalp allotransplantation presents minimal surgical difficulty, the procedure is as complex as any organ transplantation due to the issues related to immunity. First, serological screening tests and $\mathrm{ABO}$ blood typing need to be performed to assess immune compatibility between donors and recipients. In addition, donor-recipient compatibility must be evaluated for age,

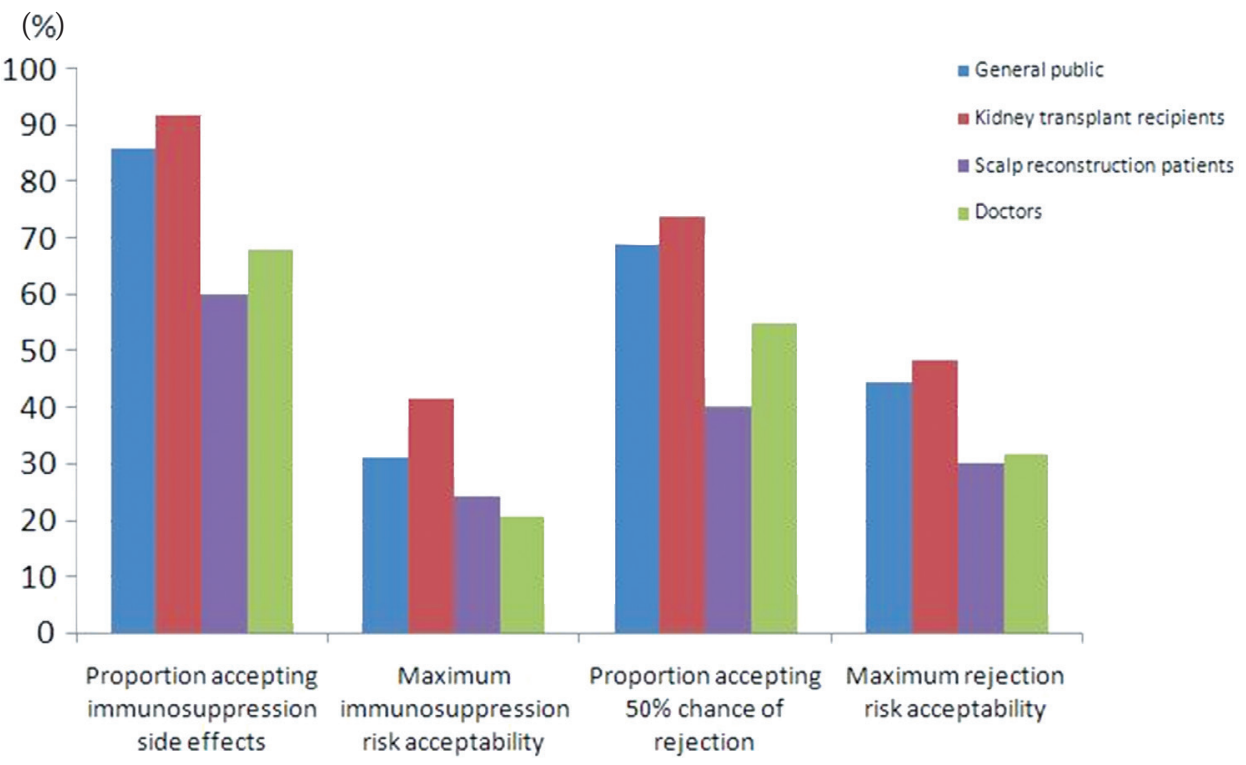

Fig. 5. Risk acceptance in scalp allotransplantation. The ability to accept risk (general public, kidney transplant recipients, postscalp reconstruction patients group, and doctors) of immunosuppression and tissue rejection in scalp allotransplantation. 


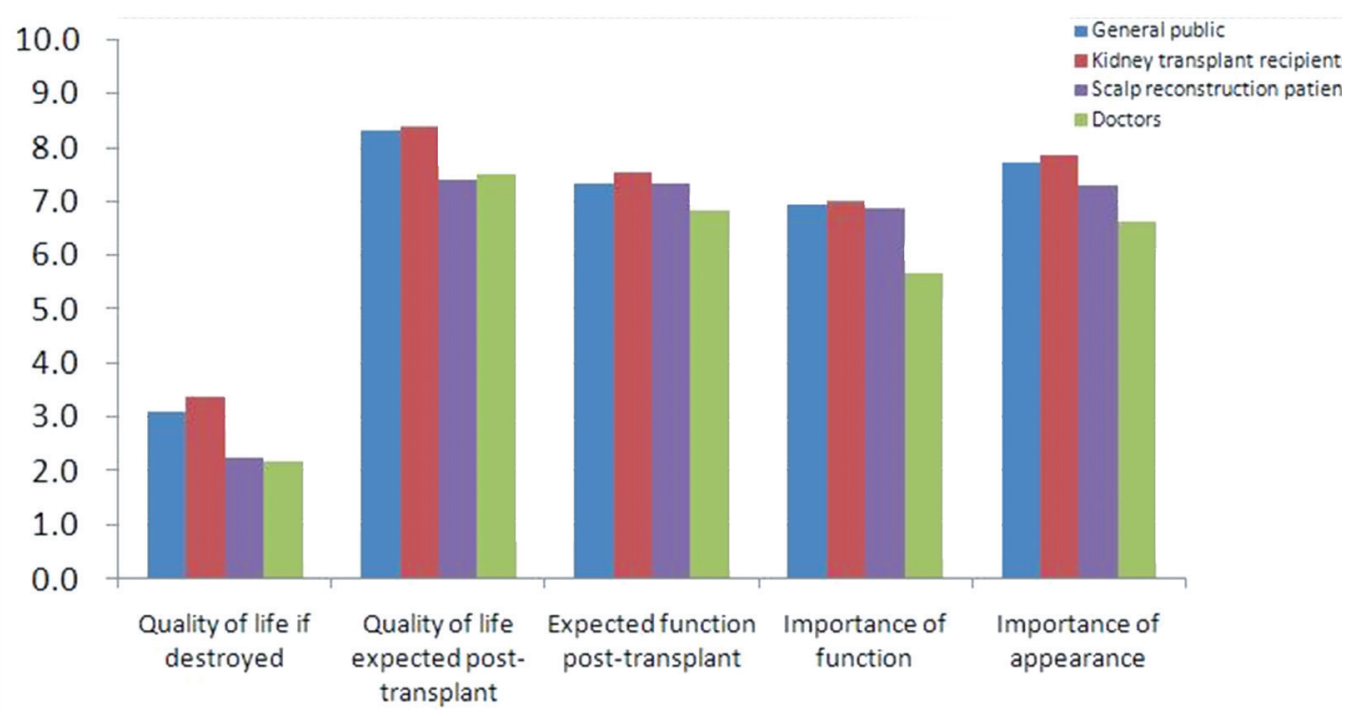

Fig. 6. Expectations in scalp allotransplantation. The expectations (general public, kidney transplant recipients, post-scalp reconstruction patients group, and doctors) of quality of life and the functional and aesthetic out $า$ comes in scalp allotransplantation.

skin tone, gender, race, and tissues dimensions. If a compatible donor is found, the composite tissue must be obtained, transported, and transplanted into the recipient while minimizing ischemic time. Transplantations requires intensive care by a multitude of healthcare personnel during the early postoperative period, including monitoring of the blood flow in and out of the transplanted organ. Tissue rejection can occur despite adequate immunosuppressive regimen, which may necessitate removal of the graft. No episode of acute rejection has been reported for the two cases of scalp allotransplantation, but recipients must remain on lifelong immunosuppressive therapy and need to be carefully monitored for occurrence of chronic graft versus host disease.

Compared to solid organ transplantation, scalp allotransplantation is challenged by a different set of problems. First, scalp transplant does not extend life, which raises ethical questions for patients whose life may be shortened due to immunosuppressive management [9]. Second, it is difficult to find a donor due to the cultural issues surrounding the procurement of scalp. A third difficulty is the selection of a recipient with good compliance for a successful scalp allotransplantation. The recipient will need to undergo screening for clear understanding of the risks and bene- fits through psychiatric evaluations, such as for schizophrenia, and an IQ test [10].

A "frame of reference" can be defined as a set of ideas, beliefs, and/or views that is used for predicting a conclusion in psychological terms and for making decision. Barker et al. suggested that since such frame of references should be observed for various groups involved in transplantations (laypersons, potential patients, physicians, etc.) because those frames modify the perception of the risks and benefits of transplantation [11]. In our study, the differences in risk acceptance and expectations for scalp transplantation across the groups most likely reflect the differences in frame of reference across the four groups.

In our study, the kidney transplantation group gave acceptable scores on the survey for risk acceptance and expectations, which seemed to indicate that they already understood immunosuppressive therapy through direct experience. Such personal experiences seems to have raised the consensus threshold value for risk acceptance. It is important to note, however, that only kidney transplantation survivors were included as group members, and the results may reflect a selection bias for positive outlook.

In contrast, the general public group gave relatively higher risk 
acceptance and expectation scores, which seemed to be mainly related to a lack of practical knowledge on the subject matter. Since most of the general public has little or no in depth knowledge about scalp allotransplantation, the survey evaluators had to provide additional explanations regarding scalp allotransplantation and immunosuppression during the survey.

Participants who had received scalp reconstruction responded differently from the transplant patients and the general public. This could be because scalp reconstruction patients felt that the numerous adverse reactions of immunosuppressive therapy, risks of histoincompatibility and additional surgery could not justify their adjusted status. Though inconvenient, the patients had adjusted to covering up the unsightly portions of the scalp with wigs or hats.

Furthermore, satisfaction related to operative outcomes was a factor that was different between the kidney transplantation group and post-scalp reconstruction group. Since scalp is not a vital organ as kidney, the defect of scalp only needs to be covered with intact skin, with or without hair. On the other hand, kidney transplant surgery not only resolves the inconvenience of dialysis but also extends the lives of chronic kidney disease patients. Therefore, the kidney transplant group was more satisfied with outcomes of their treatment and may have responded more positively for this reason.

Of the 4 groups, the group consisting of physicians demonstrated the lowest levels of risk acceptance and expectations. These participants were well aware of limitations due to gaps between expectations and outcomes; thus, they seemed to consider the adverse reactions of immunosuppressive therapy and histoincompatibility as unacceptable in return for an improvement in appearance.

Limitations of this study include the constraints of survey sample groups and geographic region. The general public group in the study was comprised of individuals visiting family members in the outpatient department, who could have a chance of bias depending on the severity and types of diseases experienced by the relative. A sample from the population outside of the hospital might better represent the general population. In particular, the general public group should be selected in consideration of more varied demographic and socioeconomic status.

The present study found results, which echo findings from other international studies and that from the allotransplantation conducted within South Korea [5-7,12]. Despite differences in transplantation type and study populations, the fact that study results were similar indicates that the most influential factor could be "expectations" of the general public group with the lack of knowledge regarding subject matter. Therefore, it is important for patients to fully understand the various difficulties of scalp allotransplantation before surgery to allow each individual to evaluate whether or not the post-transplant outcomes are acceptable.

For scalp transplantation to be a viable practice in modern medicine, physicians should not solely focus on the adverse reactions of immunosuppression and low expectations, but also increase the effort on minimizing such risks and on satisfying the cosmetic or functional requirements of patients. In addition, the public should be informed against the unrealistic expectations of allotransplantation based on incorrect portrayal from popular media.

The present study investigated differencesin the risk acceptance and expectations of scalp allotransplantation in specific sub-groups of the South Korean population and has found findings similar to those of other populations.

Scalp allotransplantation is feasible operation for large scalp defect patients. However, physicians must understand the differences in risk acceptance and expectations across various groups of the lay public and recognize the advantages and disadvantages of scalp allotransplantation.

\section{REFERENCES}

1. Buncke HJ, Hoffman WY, Alpert BS, Gordon L, Stefani AE. Microvascular transplant of two free scalp flaps between identical twins. Plast Reconstr Surg 1982;70:605-9.

2. Jiang HQ, Wang Y, Hu XB, Li YS, Li JS. Composite tissue allograft transplantation of cephalocervical skin flap and two ears. Plast Reconstr Surg 2005;115:31e-5e.

3. Barker JH, Vossen M, Banis JC Jr. The technical, immunological and ethical feasibility of face transplantation. J Surg 2004;2:8-12.

4. Shapiro PA, Kornfeld DS. Psychiatric aspects of head and neck cancer surgery. Psychiatr Clin North Am 1987;10:87-100. 
5. Park SR, Kim JH, Hwang JH, Kim KS, Lee SY. Investigation of risk acceptance and expectations in facial allotransplantation. J Korean Soc Plast Reconstr Surg 2010;37:555-60.

6. Lee DH, Kim JH, Hwang JH, Kim KS, Lee SY. Investigation of risk acceptance and expectations in hand and foot allotransplantations. J Korean Soc Plast Reconstr Surg 2011;38:427-37.

7. Jo HK, Park JW, Hwang JH, Kim KS, Lee SY, Shin JH. Risk acceptance and expectations of laryngeal allotransplantation. Arch Plast Surg 2014;41:505-12.

8. Kim HM, Kim JH, Hwang JH, Kim KS, Lee SY. Evaluation of reliability and validity of the Louisville instrument for transplantation (LIFT) in Korean population. J Korean Soc Plast Reconstr Surg 2011;38:245-50.
9. Siemionow M, Ozmen S, Demir Y. Prospects for facial allograft transplantation in humans. Plast Reconstr Surg 2004;113:1421-8.

10. Hui-Chou HG, Nam AJ, Rodriguez ED. Clinical facial composite tissue allotransplantation: a review of the first four global experiences and future implications. Plast Reconstr Surg 2010;125:538-46.

11. Barker JH, Furr A, Cunningham M, Grossi F, Vasilic D, Storey B, et al. Investigation of risk acceptance in facial transplantation. Plast Reconstr Surg 2006;118:663-70.

12. Brouha P, Naidu D, Cunningham M, Furr A, Majzoub R, Grossi FV, et al. Risk acceptance in composite-tissue allotransplantation reconstructive procedures. Microsurgery 2006;26:144-9. 